\title{
Cross-correlation redshift calibration without spectroscopic calibration samples in DES Science Verification Data
}
C. Davis, ${ }^{1 \star}$ E. Rozo, ${ }^{8}$ A. Roodman,,${ }^{1,7}$ A. Alarcon, ${ }^{2}$ R. Cawthon, ${ }^{3}$ M. Gatti, ${ }^{4}$ H. Lin, ${ }^{5}$ R. Miquel, ${ }^{4,6}$ E. S. Rykoff, ${ }^{1,7}$ M. A. Troxel, $,{ }^{9}, 10$ P. Vielzeuf, ${ }^{4}$ T. M. C. Abbott, ${ }^{11}$ F. B. Abdalla, ${ }^{12,13}$ S. Allam,${ }^{5}$ J. Annis, ${ }^{5}$ K. Bechtol, ${ }^{14}$ A. Benoit-Lévy, ${ }^{12,15,16}$ E. Bertin, ${ }^{15,16}$ D. Brooks, ${ }^{12}$ E. Buckley-Geer, ${ }^{5}$ D. L. Burke, ${ }^{1,7}$ A. Carnero Rosell, ${ }^{17,18}$ M. Carrasco Kind, ${ }^{19,20}$ J. Carretero, ${ }^{4}$ F. J. Castander, ${ }^{2}$ M. Crocce,${ }^{2}$ C. E. Cunha, ${ }^{1}$ C. B. D'Andrea, ${ }^{21}$ L. N. da Costa, ${ }^{17,18}$ S. Desai, ${ }^{22}$ H. T. Diehl, ${ }^{5}$ P. Doel, ${ }^{12}$ A. Drlica-Wagner, ${ }^{5}$ A. Fausti Neto, ${ }^{17}$ B. Flaugher,${ }^{5}$ P. Fosalba, ${ }^{2}$ J. Frieman, $, 3,5$ J. García-Bellido, ${ }^{23}$ E. Gaztanaga, ${ }^{2}$ D. W. Gerdes, ${ }^{24,25}$ T. Giannantonio, ${ }^{26,27,28}$ D. Gruen, ${ }^{1,7}$ R. A. Gruendl, ${ }^{19,20}$ G. Gutierrez, ${ }^{5}$ K. Honscheid, ${ }^{9,}{ }^{10}$ B. Jain, ${ }^{21}$ D. J. James, ${ }^{29,11}$ T. Jeltema, ${ }^{30}$ E. Krause, ${ }^{1}$ K. Kuehn, ${ }^{31}$ S. Kuhlmann, ${ }^{32}$ N. Kuropatkin, ${ }^{5}$ O. Lahav, ${ }^{12}$ T. S. Li, ${ }^{5}$ M. Lima, ${ }^{17,33}$ M. March, ${ }^{21}$ J. L. Marshall, ${ }^{34}$ P. Martini,,${ }^{9,35}$ P. Melchior, ${ }^{36}$ R. L. C. Ogando, ${ }^{17,18}$ A. A. Plazas, ${ }^{37}$ A. K. Romer ${ }^{38}$ E. Sanchez, ${ }^{39}$ V. Scarpine, ${ }^{5}$ R. Schindler, ${ }^{7}$ M. Schubnell, ${ }^{25}$ I. Sevilla-Noarbe, ${ }^{39}$ M. Smith, ${ }^{40}$ M. Soares-Santos, ${ }^{5}$ F. Sobreira, $,{ }^{41}, 17$ E. Suchyta, ${ }^{42}$ M. E. C. Swanson, ${ }^{20}$ G. Tarle, ${ }^{25}$ D. Thomas, ${ }^{43}$ V. Vikram, ${ }^{32}$ A. R. Walker ${ }^{11}$ and R. H. Wechsler ${ }^{1,7,44}$ (DES Collaboration)

Affiliations are listed at the end of the paper

Accepted 2018 March 22. Received 2018 February 2; in original form 2017 July 25

\begin{abstract}
Galaxy cross-correlations with high-fidelity redshift samples hold the potential to precisely calibrate systematic photometric redshift uncertainties arising from the unavailability of complete and representative training and validation samples of galaxies. However, application of this technique in the Dark Energy Survey (DES) is hampered by the relatively low number density, small area, and modest redshift overlap between photometric and spectroscopic samples. We propose instead using photometric catalogues with reliable photometric redshifts for photo- $z$ calibration via cross-correlations. We verify the viability of our proposal using redMaPPer clusters from the Sloan Digital Sky Survey (SDSS) to successfully recover the redshift distribution of SDSS spectroscopic galaxies. We demonstrate how to combine photo- $z$ with cross-correlation data to calibrate photometric redshift biases while marginalizing over possible clustering bias evolution in either the calibration or unknown photometric samples. We apply our method to DES Science Verification (DES SV) data in order to constrain the photometric redshift distribution of a galaxy sample selected for weak lensing studies, constraining the mean of the tomographic redshift distributions to a statistical uncertainty of $\Delta z \sim \pm 0.01$. We forecast that our proposal can, in principle, control photometric redshift
\end{abstract}


uncertainties in DES weak lensing experiments at a level near the intrinsic statistical noise of the experiment over the range of redshifts where redMaPPer clusters are available. Our results provide strong motivation to launch a programme to fully characterize the systematic errors from bias evolution and photo- $z$ shapes in our calibration procedure.

Key words: galaxies: clusters: general-galaxies: distances and redshifts.

\section{INTRODUCTION}

The determination of the photometric redshift distribution of a given source population is fraught with difficulties. For instance, the two primary methods for determining the redshift distribution of photometric objects - template fitting and machine learning - must both confront a critical difficulty: the spectroscopy available for training, calibration, and validation of photometric redshift techniques is rarely a representative of the magnitude and colour-space distribution of all survey galaxies. It is possible to mitigate this problem by weighting spectroscopic galaxies such that they better represent the photometric properties of the whole photometric survey (Lima et al. 2008; Cunha et al. 2009; Sánchez et al. 2014; Bonnett et al. 2016, Hoyle et al. in preparation). However, for this method to be effective, the training set must be complete relative to the photometric data, such that it densely covers and spans the same space of relevant photometric observables as the full survey. This is difficult to achieve in regions that lack spectroscopic data, particularly at high redshifts. Similarly, available templates may not span the full colour-redshift-space (Bonnett et al. 2016) of the galaxies of interest. This problem tends to be particularly acute for the faint galaxies which make up the majority of the sources used for weak gravitational lensing. In order to precisely and accurately determine the dark energy equation of state from photometric weak lensing and large-scale structure measurements, it is vital to precisely characterize the redshift distributions of the tomographic redshift bins into which the galaxies are split. Bonnett et al. (2016) find that photometric redshifts biases must be controlled at the $\sim 0.003$ level in order for the Dark Energy Survey (DES) $5000 \mathrm{deg}^{2}$ survey to not be limited by photometric redshift uncertainties. While 'photo- $z$ ' methods have made considerable progress towards meeting these requirements, current performance falls short of this goal. As such, any method with the potential to further improve this calibration is of great interest as a possible way to reduce, and perhaps even eliminate, the photo- $z$ systematics floor of photometric surveys like the DES.

Schneider et al. (2006) first proposed using cross-correlations between galaxies binned by photo- $z$ to constrain photo- $z$ errors. Newman (2008) then demonstrated that by cross-correlating a sample of photometric galaxies with an unknown redshift distribution with thin redshift slices of a spectroscopic galaxy sample one could recover the redshift distribution of the photometric galaxy sample. This method was improved by Matthews \& Newman (2010) using an iterative technique to account for the evolution in galaxy clustering bias. Several others have tested the method on $N$-body simulations with promising results, including various methods for further improving and refining the cross-correlation method (Schulz 2010; Matthews \& Newman 2012; McQuinn \& White 2013; Schmidt et al. 2013; McLeod, Balan \& Abdalla 2017; van Daalen \& White 2017; Scottez et al. 2018). The method has also been applied to data: Benjamin et al. (2010) used cross-correlations to measure the degree of artificial contamination in tomographic redshift bins and applied the technique to the Canada-France-Hawaii Telescope Legacy Survey (CFHTLS), and Ménard et al. (2013) used clustering measurements on both linear and modestly non-linear scales to characterize the redshift distributions of (Sloan Digital Sky Survey) SDSS, WISE, and FIRST galaxies.

More recently, Rahman et al. (2015) combined clustering information with photometry to show how the cross-correlation method can recover the redshift probability distribution of an individual galaxy. Schmidt et al. (2015) cross-correlated Planck High Frequency Instrument maps against SDSS quasars to estimate the distribution of the cosmic infrared background. Rahman et al. (2016) mapped the relation between galaxy colour and redshift by using cross-correlations instead of spectral energy distribution templates, and Scottez et al. (2016) apply the same estimations to VIPERS. Sun, Zhan \& Tao (2015) extend cross-correlations to the Fourier domain by examining how galaxy angular power spectra can determine the mean redshift to percent precision. Lee et al. (2016) use integer linear programming to optimize crossand autocorrelations, demonstrating that it is possible to assign individual galaxies to redshift bins via clustering signals alone. Choi et al. (2016) used cross-correlations to check the validity of using summed $p(z)$ to determine galaxy redshift distributions in both CFHTLS and the Red-sequence Cluster Lensing Survey.

Most recently, Hildebrandt et al. (2017) used cross-correlation methods to validate the photometric redshift distribution of source galaxies in the Kilo Degree Survey via the open source code ThewizZ described in Morrison et al. (2017). Johnson et al. (2017) extended the quadratic estimator from McQuinn \& White (2013) and found qualitative agreement with the results from Hildebrandt et al. (2017). Importantly, those efforts were hampered by the low number of spectroscopic galaxies available for photo- $z$ calibration via cross-correlations across a broad redshift range. This relative lack of spectroscopic calibration samples for cross-correlation studies is a serious obstacle to the realization of the promise that these methods hold.

One solution to the relative paucity of spectroscopic galaxies in the footprint of these wide-field optical photometric surveys is to use other objects which have reasonably precise redshifts but which are far more numerous. Indeed, Schneider et al. (2006) pointed out that the cross-correlation procedure benefits greatly from samples of objects whose photo- $z$ can be estimated unusually well. One example for such a class of objects is redMaPPer galaxy clusters described in Rykoff et al. (2014), who present a red sequence cluster finder that produces objects with nearly Gaussian estimated redshifts and scatter $\sigma_{z} /(1+z) \sim 0.01$. The method relies on a small set of spectroscopic objects for training, but can then be used to find objects over the full survey footprint to much fainter magnitudes. Importantly, while these objects are rare, the complete overlap between these photometrically selected objects and the galaxies that are to be calibrated means that the cross-correlation signal can be measured with high signal-to-noise. This makes them a natural candidate for calibrating redshift distributions in photometric wide-field surveys. Note, however, that such cross-correlation measurements are by necessity limited to the redshift range over which the red sequence is well calibrated. 
In this paper, we examine how well the cross-correlation method performs when, instead of spectroscopic galaxies, objects with accurate photometric redshifts are used as the reference sample. We wish to examine the following questions:

(i) How well do cross-correlation methods with nonspectroscopic reference samples perform in comparison with spectroscopic reference samples?

(ii) How can we properly combine photo- $z$ and cross-correlation information to minimize the noise inherent to cross-correlation methods while reducing the redshift biases from standard photo$z$ methods?

(iii) How does using non-spectroscopic reference samples to constrain the redshift distributions of galaxies impact cosmological parameter estimation?

This paper is organized as follows. In Section 2, we present the data sets used in this study. In Section 3, we review the theory behind the cross-correlation method and present our method for calculating redshift distributions from cross-correlations. In Section 4, we present the performance of cross-correlations when using redMaPPer clusters as reference objects, and compare our results to those obtained when using SDSS spectroscopic galaxies as reference. We also examine the impact of using different weighting functions and integration ranges on the accuracy and precision of the recovered redshift distributions. In Section 5, we apply our method to the Dark Energy Survey Science Verification (DES SV) data set, and examine how we can use the cross-correlation method to determine the redshift bias in photometric redshift methods. In Section 6, we forecast the performance of using the cross-correlation method to constrain cosmological parameters with the Dark Energy Survey Year Five data. We wrap up in Section 7 and discuss potential future applications of this method.

Throughout this paper, we assume a WMAP9 cosmology $\left(\Omega_{\mathrm{m}}=0.286\right)$ and report distances in $h^{-1} \mathrm{Mpc}$. We find that the choice of cosmology has a negligible impact on our results (Hinshaw et al. 2013).

\section{DATA SETS}

Our analysis relies on four catalogues drawn from two galaxy surveys, the SDSS and the DES SV. Each of these data sets and the corresponding catalogues are detailed below.

\subsection{SDSS spectroscopic galaxy samples}

The SDSS spectroscopic galaxy sample consists of the LOWZ and CMASS galaxy samples from the Baryon Oscillation Spectroscopic Survey (BOSS) (Alam et al. 2015). BOSS obtained data over $9376 \mathrm{deg}^{2}$ divided into two regions in the Northern and Southern Galactic Caps. The LOWZ sample is a set of galaxies uniformly targeted for large-scale structure studies in a relatively lowredshift range $(z<0.43)$. The CMASS sample is a set of galaxies over the range $(0.43<z<0.7)$ designed to create an approximately volume-limited sample in stellar mass. Both catalogues come with 'randoms', catalogues that reflect the footprints and selection functions of the two surveys. For our purposes here, it is sufficient to simply combine the two catalogues over their full redshift ranges, which we shall collectively refer to as the SDSS catalogue.

\section{2 redMaPPer}

redMaPPer is a red sequence cluster finder originally developed within the context of the SDSS (Rykoff et al. 2014; Rozo et al. 2015b). The red sequence is the empirical relation that early-type galaxies in rich clusters lie along a linear colour-magnitude relation with small scatter (Bower, Lucey \& Ellis 1992; Bell et al. 2004). redMaPPer iteratively self-trains a model of the red sequence based on sparse spectroscopic data, and then uses this model to identify clusters of red sequence galaxies, and to estimate the photometric redshift of the resulting clusters. The algorithm has been extensively tested and validated (Rozo et al. 2015a). In addition to the SDSS catalogue, we use the redMaPPer cluster catalogue resulting from the application of the redMaPPer algorithm to the DES SV data (Rykoff et al. 2016). While the redMaPPer cluster catalogue from SDSS is therefore different from the one used in DES SV, in that the algorithm has been applied to a different set of galaxies, the number density and the mass-richness relations are similar, and most importantly, the performance in photo- $z$ is the same. These catalogues come with their own associated 'randoms' which reflect the footprint and the ability to select a redMaPPer cluster of some richness $\lambda$ at a given location. We have chosen clusters with $\lambda>5$. For our purposes, the most important aspect of the redMaPPer cluster catalogues its exquisite redshift performance: cluster photo-z's are both accurate (photo- $z$ redshift biases are at the 0.005 level or less) and precise (photo- $z$ scatter is $\sigma_{z} /(1+z) \sim 0.01$ ) with a $4 \sigma$ outlier fraction of at most 3 per cent. As such, the redMaPPer clusters are excellent candidates for photometric reference samples when attempting to perform photo- $z$ calibration via cross-correlation.

Unfortunately, the redMaPPer clusters do not span the full redshift range of the samples we consider in this paper. In SDSS, the redshift range of the redMaPPer clusters used is $0.1<z<0.56$, while in DES SV we take advantage of the greater depth and look at clusters in the range $0.2<z<0.85$. The greater redshift reach in the DES SV region is due to the greater depth of the photometric data. In contrast, the SDSS spectroscopic galaxies extend to $z<0.7$, while the source galaxies in DES SV can have redshifts of $z>1$.

\subsection{DES SV}

The DES is an ongoing $5000 \mathrm{deg}^{2}$ photometric survey in the grizY bands performed with the Dark Energy Camera (DECam; Flaugher et al. 2015). Before the beginning of the survey, the DES conducted an $\sim 250 \mathrm{deg}^{2}$ 'Science Verification' (SV) survey. The main portion of the SV footprint, used in this paper, covers $139 \mathrm{deg}^{2}$ in the range $65<$ R.A. $<93$ and $-60<$ Decl. $<-42$. The region is dubbed South Pole Telescope East (SPTE) because of its location and overlap with the South Pole Telescope survey area (SPT; Carlstrom et al. 2011). SPTE was observed with 2-10 tilings in each of the griz filters. In addition, the DES observes 10 Supernova fields every 5-7d, each of which covers a single DECam 2.2 deg-wide field of view. The median depth of the SV survey (defined as $10 \sigma$ detections for extended sources) is $g=24.0, r=23.8 i=23.0, z=22.3$, and $Y=20.8$.

The DES SV data were processed by the DES Data Management infrastructure (Morganson et al. 2018, see also Sevilla et al. 2011; Desai et al. 2012). We used SExtractor (Bertin \& Arnouts 1996; Bertin 2011) to detect photometric sources based on a 'chi-squared' co-add of $r, i$, and $z$ images obtained with SWarp (Bertin 2010). The catalogue was then further refined to produce the DES 'SVA1 Gold' 
catalogue. ${ }^{1}$ Galaxy magnitudes and colours are computed via the SExtractor MAG_AUTO quantity. This SVA1 Gold catalogues the fundamental input to the construction of the DES SV NGMIX galaxy sample and the DES SV redMaPPer cluster catalogue.

\subsection{NGMIX}

From the SVA1 Gold catalogue, we examine a subsample of galaxies used for cosmic shear measurements. ${ }^{2}$ The NGMIX pipeline (Sheldon 2014) estimates the shapes of the galaxies in the SVA1 catalogue. The subsample is then selected by cutting objects with very low surface brightnesses and small sizes, and choosing only objects with reasonable colours $(-1<g-r<4$ and $-1<i-z<4)$. NGMIX represents galaxies as sums of Gaussians (Hogg \& Lang 2013). The same model shape is fitted simultaneously across multiple exposures in the riz bands using Markov Chain Monte Carlo techniques applied to a full likelihood which forward models the galaxy by convolving with the exposure PSF. The final effective source number density of the NGMIX catalogue is $\sim 6.1$ galaxies per square arcminute (Becker et al. 2016). The shape systematics in the NGMIX catalogue are tangential to this paper to the extent that they do not imprint a spatial correlation on the footprint. However, interested readers should look to Jarvis et al. (2016) for a thorough examination of shape systematics.

Galaxy photo-z's for the NGMIX catalogue are estimated using four different photometric redshift algorithms: the template-based algorithm BPZ (Benítez 1999; Coe et al. 2006), and the machine learning algorithms SkyNet (Graff et al. 2014; Bonnett 2015), TPZ (Carrasco Kind \& Brunner 2013, 2014), and ANNZ2 (Sadeh, Abdalla \& Lahav 2016). Extensive testing of the algorithms was carried out in Sánchez et al. (2014) and Bonnett et al. (2016), to which we refer the reader for further detail on the algorithms. We do not include the calibration offset to BPZ that was measured in Bonnett et al. (2016). For our purposes, the key point is that each of these algorithms returns a redshift probability distribution $P(z)$ for each galaxy, the sum of which can be used as a (biased) estimator for the redshift distribution of the galaxies under consideration (Asorey et al. 2016).

\section{THEORY AND METHODS}

We outline the theory in this section and present our estimator. The approach presented here is based on Ménard et al. (2013). We repeat much of the argument here, though the focus and presentation are somewhat different. Our goal is not to be repetitious, but to simply present an alternative but fully equivalent discussion.

Let $\rho$ be the comoving galaxy density. In a flat universe, the corresponding galaxy density per unit angular area per unit redshift $\mathrm{d}^{2} N / \mathrm{d} \Omega \mathrm{d} z$ is given by

$\frac{\mathrm{d}^{2} N}{\mathrm{~d} \Omega \mathrm{d} z}(\mathrm{~d} \Omega \mathrm{d} z)=\rho(z) \chi^{2}(z) \frac{\mathrm{d} \chi}{\mathrm{d} z} \mathrm{~d} \Omega \mathrm{d} z=\rho(z) \chi^{2}(z) c H^{-1}(z) \mathrm{d} \Omega \mathrm{d} z$,

\footnotetext{
${ }^{1}$ https://des.ncsa.illinois.edu/releases/sva1

${ }^{2}$ The DES SV cosmic shear analysis also used a second shape measurement pipeline, IM3 SHAPE (Zuntz et al. 2013). Since we are only interested in verifying the feasibility of the proposed cross-correlation measurement, in this work we have limited ourselves to the NGMIX sample because of its higher space density.
}

where $\chi$ is the comoving distance and $H$ is the Hubble parameter. One has then

$v \equiv \frac{\mathrm{d}^{2} N}{\mathrm{~d} \Omega \mathrm{d} z}=\bar{\rho}(z) \chi^{2}(z) c H^{-1}(z)[1+\delta(\chi(z) \theta, \chi(z))]$,

where $v$ is the angular galaxy density per unit redshift and per unit angular area, $\delta$ is the fractional matter overdensity relative to the mean density, and $\theta$ is the angular separation.

Let $n(\theta)=\mathrm{d} N / \mathrm{d} \Omega$ be the projected galaxy density. One has

$n(\theta)=\int \mathrm{d} z \bar{\rho}(z) \chi^{2}(z) c H^{-1}(z)[1+\delta(\chi(z) \theta, \chi(z))]$.

The mean galaxy density is simply

$\bar{n}=\int \mathrm{d} z \bar{\rho}(z) \chi^{2}(z) c H^{-1}(z)$,

while the redshift distribution of the galaxies $\phi(z)$ is defined such that $\bar{n} \phi(z)$ is the mean galaxy density per unit redshift, i.e.

$\bar{n} \phi(z)=\bar{v}=\bar{\rho}(z) \chi^{2}(z) c H^{-1}(z)$.

We can therefore write

$n(\theta)=\bar{n} \int \mathrm{d} z \phi(z)[1+\delta(\chi(z) \theta, \chi(z))]$,

where $\phi(z)$ integrates to unity. Setting $n(\theta)=\bar{n}(1+\Delta)$, we see that

$\Delta(\theta)=\int \mathrm{d} z \phi(z) \delta(\chi \theta, \chi)$.

Consider now two galaxy samples, one of which has known redshifts, which we refer to as the reference sample with a subscript 'ref', and one which has an unknown redshift distribution, which we refer to as the unknown sample with a subscript ' $u$ '. We wish to consider the cross-correlation between the unknown sample and reference galaxies within a narrow redshift bin. The angular crosscorrelation between the reference and unknown samples is therefore

$w(\theta)=\left\langle\Delta_{\mathrm{u}} \Delta_{\mathrm{ref}}\right\rangle(\theta)$

$$
=\int \mathrm{d} z \mathrm{~d} z^{\prime} \phi_{\mathrm{u}}(z) \phi_{\mathrm{ref}}\left(z^{\prime}\right) b_{\mathrm{u}}(\theta, z) b_{\mathrm{ref}}\left(\theta, z^{\prime}\right) \xi\left(\chi \theta, \chi ; \chi^{\prime} \theta, \chi^{\prime}\right)
$$

where $\xi$ is the matter-matter correlation function $\left\langle\delta(\chi \theta, \chi) \delta\left(\chi^{\prime} \theta\right.\right.$, $\left.\left.\chi^{\prime}\right)\right\rangle$ and we allow the galaxy clustering bias $b$ to have both redshift and scale dependence for some separation $\theta$ and redshift $z$. Consider the case $\phi_{\text {ref }}(z)=\delta\left(z-z_{\text {ref }}\right)$, where $z_{\text {ref }}$ is some reference redshift. This is equivalent to selecting a reference sample in an infinitely narrow redshift slice. We have then

$w\left(\theta, z_{\text {ref }}\right)=b_{\text {ref }}\left(\theta, z_{\text {ref }}\right) \int \mathrm{d} z \phi_{\mathrm{u}}(z) b_{\mathrm{u}}(\theta, z) \xi\left(\chi \theta, \chi ; \chi^{\prime} \theta, \chi^{\prime}\right)$.

Now, $\xi$ is zero unless $\chi \sim \chi^{\prime}=\chi\left(z_{\text {ref }}\right)$. Using a flat sky approximation, and adopting the origin at a reference galaxy when measuring the angular separation, we find

$$
\begin{aligned}
w\left(\theta, z_{\text {ref }}\right)= & b_{\text {ref }}\left(\theta, z_{\text {ref }}\right) \int \mathrm{d} z \phi_{\mathrm{u}}(z) b_{\mathrm{u}}(\theta, z) \\
& \times \xi\left(\left[\Delta \chi^{2}\left(z, z_{\text {ref }}\right)+\chi\left(z_{\text {ref }}\right)^{2} \theta^{2}\right]^{1 / 2} ; z_{\text {ref }}\right),
\end{aligned}
$$

where $\Delta \chi=\chi(z)-\chi\left(z_{\text {ref }}\right)$. We assume $\xi$ varies much more quickly than $\phi_{\mathrm{u}}$ or $b_{\mathrm{u}}$. Since $\xi$ is non-zero only over a small redshift range 
$z \sim z_{\text {ref }}$, we arrive at

$$
\begin{aligned}
w\left(\theta, z_{\text {ref }}\right)= & \phi_{\mathrm{u}}\left(z_{\text {ref }}\right) b_{\mathrm{u}}\left(\theta, z_{\text {ref }}\right) b_{\text {ref }}\left(\theta, z_{\text {ref }}\right) \\
& \times \int \mathrm{d} z \xi\left(\left[\Delta \chi^{2}+\chi\left(z_{\text {ref }}\right)^{2} \theta^{2}\right]^{1 / 2} ; z_{\text {ref }}\right) .
\end{aligned}
$$

It is useful to rephrase the correlation function in terms of the physical transverse separation $r=D_{A} \theta=(1+z)^{-1} \chi \theta$. The integral of the correlation function is simply the projected correlation function for matter

$w_{\mathrm{mm}}\left(r, z_{\text {ref }}\right)=\int \mathrm{d} z \xi\left(\left[\left(\chi(z)-\chi\left(z_{\text {ref }}\right)\right)^{2}+(1+z)^{2} r^{2}\right]^{1 / 2} ; z_{\text {ref }}\right)$.

Again, note we have opted to utilize physical units for $r$. Plugging in we arrive at

$w\left(r, z_{\text {ref }}\right)=\phi_{\mathrm{u}}\left(z_{\text {ref }}\right) b_{\mathrm{u}}\left(r, z_{\text {ref }}\right) b_{\text {ref }}\left(r, z_{\text {ref }}\right) w_{\mathrm{mm}}\left(r, z_{\text {ref }}\right)$.

If we define the growth $G\left(r, z_{\text {ref }}\right)$ relative to some arbitrary redshift $z_{0}$ via

$w_{\mathrm{mm}}\left(r, z_{\text {ref }}\right)=G\left(r, z_{\text {ref }}\right) w_{\mathrm{mm}}\left(r, z_{0}\right)$,

our final expression becomes

$w\left(r, z_{\text {ref }}\right)=\phi_{\mathrm{u}}\left(z_{\text {ref }}\right) b_{\mathrm{u}}\left(r, z_{\text {ref }}\right) b_{\text {ref }}\left(r, z_{\text {ref }}\right) G\left(r, z_{\text {ref }}\right) w_{\mathrm{mm}}\left(r, z_{0}\right)$

Note $G$ is not necessarily the linear growth factor, and in fact, $G$ can depend on the length-scale $r$. We collect $b_{\mathrm{u}}\left(r, z_{\text {ref }}\right), b_{\text {ref }}\left(r, z_{\text {ref }}\right)$, and $G\left(r, z_{\text {ref }}\right)$ into a single source function $f\left(r, z_{\text {ref }}\right)$ to write

$w\left(r, z_{\mathrm{ref}}\right)=\phi_{\mathrm{u}}\left(z_{\mathrm{ref}}\right) f\left(r, z_{\mathrm{ref}}\right) w_{\mathrm{mm}}(r)$,

where we have made the evaluation $z=z_{0}$ of $w_{\mathrm{mm}}$ implicit. We will address how we handle the function $f\left(r, z_{\text {ref }}\right)$ momentarily.

We measure the angular correlation function by counting the number of pairs between our unknown and reference data $D_{u} D_{r}$ separated over a range of scales $r_{\min }$ to $r_{\max }$ weighted by some function $W(r)$. We take $W(r)$ to be a power law, $W(r) \propto r^{\alpha}$. We discuss how we select the value $\alpha$ and the radial range $r_{\min }$ to $r_{\max }$ in Sections 4 and 5 below.

Given the true angular correlation $w$, the number of unknown reference pairs is

$\mathrm{D}_{\mathrm{u}} \mathrm{D}_{\mathrm{r}}(z)=n_{\mathrm{u}} n_{\mathrm{r}} A_{\text {survey }} A_{\text {shell }} \int_{r_{\text {min }}}^{r_{\max }} \mathrm{d} r W(r)[1+w(r, z)]$,

where $n_{\mathrm{u}}$ and $n_{\mathrm{r}}$ are the number densities of the unknown and reference samples, $A_{\text {survey }}$ is the area of the survey, and $A_{\text {shell }}$ is the area of the shell over which the correlation function is computed. Similar expressions hold for random point combinations $R_{\mathrm{u}} R_{\mathrm{r}}, \mathrm{D}_{\mathrm{u}} \mathrm{R}_{\mathrm{r}}$, and $\mathrm{R}_{\mathrm{u}} \mathrm{D}_{\mathrm{r}}$, only with $w=0$. It is easy to check that the Landy \& Szalay (1993) estimator with these pair counts results in an estimate of the weighted average correlation function $\hat{w}$. Of course, one could compute the correlation function in narrow bins first, and then average, but the two procedures are not equivalent, and we expect weighting the pairs before averaging results in a more stable estimator since the averaging is done before one takes the ratio of the data. Our estimator for the weighted average correlation function is

$\hat{w}(z)=\frac{\mathrm{D}_{\mathrm{u}} \mathrm{D}_{\mathrm{r}}(z) N_{\mathrm{R}_{\mathrm{u}}} N_{\mathrm{R}_{\mathrm{r}}}}{\mathrm{R}_{\mathrm{u}} \mathrm{R}_{\mathrm{r}}(z) N_{\mathrm{D}_{\mathrm{u}}} N_{\mathrm{D}_{\mathrm{r}}}}-\frac{\mathrm{D}_{\mathrm{u}} \mathrm{R}_{\mathrm{r}}(z) N_{\mathrm{R}_{\mathrm{u}}}}{\mathrm{R}_{\mathrm{u}} \mathrm{R}_{\mathrm{r}}(z) N_{\mathrm{D}_{\mathrm{u}}}}-\frac{\mathrm{R}_{\mathrm{u}} \mathrm{D}_{\mathrm{r}}(z) N_{\mathrm{R}_{\mathrm{r}}}}{\mathrm{R}_{\mathrm{u}} \mathrm{R}_{\mathrm{r}}(z) N_{\mathrm{D}_{\mathrm{r}}}}+1$,

where $N_{\mathrm{D}_{u}}$ is the number of unknown objects, and similar definitions hold for $N_{\mathrm{D}_{\mathrm{r}}}, N_{\mathrm{R}_{\mathrm{u}}}, N_{\mathrm{R}_{\mathrm{r}}}$.
In practice, the NGMIX selection is not uniform in space, and its spatial structure has not been characterized. Consequently, a random catalogue for the NGMIX galaxies does not exist. When the unknown sample does not have a well-characterized random catalogue, we rely instead on the estimator

$\hat{w}_{\mathrm{noRu}}(z)=\frac{\mathrm{D}_{\mathrm{u}} \mathrm{D}_{\mathrm{r}}(z) N_{\mathrm{R}_{\mathrm{r}}}}{\mathrm{D}_{\mathrm{u}} \mathrm{R}_{\mathrm{r}}(z) N_{\mathrm{D}_{\mathrm{r}}}}-1$

The expectation value of both estimators in the limit of infinitely large random catalogues is

$\langle\hat{w}(z)\rangle=\phi_{\mathrm{u}}(z) \frac{\int_{r_{\min }}^{r_{\max }} \mathrm{d} r W(r) f(r, z) w_{\mathrm{mm}}(r)}{\int_{r_{\min }}^{r_{\max }} \mathrm{d} r W(r)}$.

In practice, the finite number of random points must introduce second-order corrections to our estimator. However, such corrections do not appear to have any significant effect on our results given the large number of randoms we use $(R / D \gtrsim 100)$.

We define the function $f(z)$ via

$f(z)=\frac{\int_{r_{\min }}^{r_{\max }} \mathrm{d} r W(r) f(r, z) w_{\mathrm{mm}}(r)}{\int_{r_{\min }}^{r_{\max }} \mathrm{d} r W(r)}$.

With this definition, the expectation value for $\hat{w}$ becomes

$\langle\hat{w}(z)\rangle=\phi_{\mathrm{u}}(z) f(z)$

where $f(z)$ is an unknown function that characterizes the (possibly non-linear) growth in the correlation function, and/or possibly evolving non-linearities in the correlation function. This function encompasses complicated clustering bias evolution in both the reference and the unknown samples. It could, for example, be driven by changes in the mixture of galaxy types with redshift in the unknown sample. In the absence of a strong theoretical prejudice, we adopt a simple power-law parametrization for $f(z)$,

$f(z)=f_{0}(1+z)^{\gamma}$.

With this parametrization, our final expression for the expectation value of the cross-correlation is

$\langle\hat{w}(z)\rangle=\phi_{\mathrm{u}}(z) f_{0}(1+z)^{\gamma}$.

This expression has two parameters: $\gamma$, which characterizes the redshift evolution in the correlation function (including possible non-linear effects, and $f_{0}$, which characterizes primarily nuisance deviations from unity normalization over the range of redshifts sampled by the reference sample.

Given a model for the redshift distribution $\phi_{\mathrm{u}}(z)$, which may itself depend on unknown parameters, we can recover redshift distribution through the usual $\chi^{2}$ statistic,

$\chi^{2}=\Delta w \hat{\Sigma}^{-1} \Delta w$,

where $\hat{\Sigma}$ is our estimated covariance in the observed crosscorrelation $\hat{w}$, and where

$\Delta w=\hat{w}-f_{0}(1+z)^{\gamma} \phi(z)$.

In practice, we find that parameterizing $f_{0}$ as $\mathrm{e}^{k}$ and fitting instead for $k$ improve performance.

We calculate the pairs using the code TreeCorr, ${ }^{3}$ a $\mathrm{C}$ and Python package for efficiently computing two-point and threepoint correlation functions (Jarvis, Bernstein \& Jain 2004). We estimate the covariance matrix of our estimation of $\phi_{\mathrm{u}}$ with 100 jackknife regions on the survey footprint.

\footnotetext{
${ }^{3}$ https://github.com/rmjarvis/TreeCorr
} 


\section{TESTING THE CROSS-CORRELATION METHOD WITH THE SLOAN DIGITAL SKY SURVEY}

The simplest way to test whether we can use redMaPPer clusters to measure redshift distributions through cross-correlations is to look at the performance of cross-correlations on spectroscopic galaxies. We approach this problem in two steps: first, we verify the validity of cross-correlations by determining the redshift distribution of a spectroscopic subsample of galaxies by cross-correlating it against an independent spectroscopic reference sample. Then we examine the recovery of the redshift distribution of SDSS galaxies using redMaPPer clusters as the reference sample.

We combine the CMASS and LOWZ samples and randomly split the galaxies into a reference sample containing 80 percent of the galaxies and an unknown sample containing the remaining 20 per cent. The random selection ensures that the redshift distributions of the samples are identical. The reference sample is divided according to spectroscopic redshift into bins of width $\delta z=0.02$. Pair counts of the sample and randoms are counted as described above in Section 3. The pair counts are integrated into a single scalar from $100 \mathrm{kpc}$ to $10 \mathrm{Mpc}$ and weighted by $g(R)=R^{-1}$. In order to facilitate later comparison with redMaPPer clusters, which have a more limited redshift range than the spectroscopic sample, we normalize the distribution such that its integral from $z=0.1$ to 0.56 is one. The highest redshift is set by the highest redshift for which we have enough redMaPPer clusters to obtain reasonable cross-correlation measurements. Covariances are estimated from jackknife samples over the survey footprint. We find that ignoring the bias evolution (setting $\gamma=0$ ) produces a poor fit between the clustering and spectroscopic measurements $\left(\chi^{2} /\right.$ dof $\left.=58 / 23\right)$. We fit the bias evolution model described by equation (27) and find that this brings our results into agreement $\left(\chi^{2} /\right.$ dof $\left.=25 / 21\right)$, with $\gamma=-1.7 \pm 0.5$. The results can be seen in the black triangles of Fig. 1 . These results validate our cross-correlation technique.

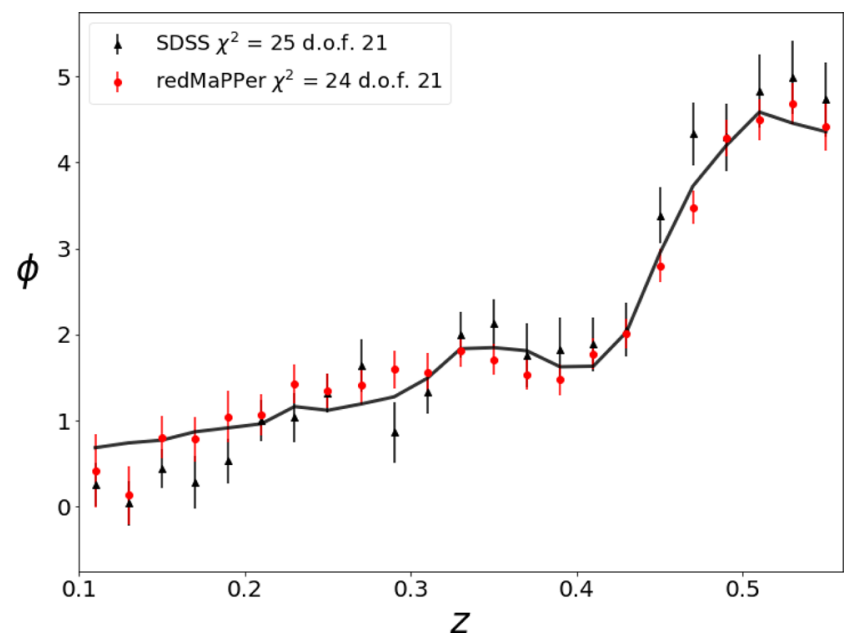

Figure 1. A comparison of the cross-correlation recovery of the SDSS redshift distribution with their spectroscopic redshifts. The solid line is the actual distribution of galaxies from spectroscopic data. The black points show the recovered SDSS redshift distribution using cross-correlation with a spectroscopic sample of SDSS galaxies as a reference sample. The red points show the corresponding redshift distributions when using redMaPPer galaxy clusters as the reference sample. Both sets of points account for the best-fitting redshift evolution in the clustering bias.
redMaPPer clusters, like all photometric algorithms, can suffer from systematic redshift biases caused by the telescope's filter set. Degeneracies in colour-magnitude space can lead to catastrophic outliers preferentially concentrating at specific redshift values. For example, Rykoff et al. (2014) saw an artificial spike near the filter transition at $z \approx 0.35$ for rich $(\lambda / S>20)$ redMaPPer clusters with spectroscopic central galaxies. Such concentrations will bias cross-correlation measurements at specific redshifts for redMaPPer clusters, but will not bias spectroscopic cross-correlations. We see no evidence in Fig. 1 for such an effect in our SDSS redMaPPer clusters, which typically have a much lower richness $(\lambda / S>5)$.

Next, we repeat the same exercise, only now the reference objects are the redMaPPer clusters, and the unknown objects are the entire SDSS CMASS and LOWZ samples. We emphasize that our goal here is to test whether we can use redMaPPer clusters to measure redshift distributions through the cross-correlation, even if our signal includes scales well within the radius of a redMaPPer cluster. We again fit the same model to the clustering signal, and find that the cross-correlation method with redMaPPer clusters as a reference sample successfully recovers the correct redshift distribution of the unknown sample within noise $\left(\chi^{2} /\right.$ dof $\left.=24 / 21\right)$, with $\gamma=-0.2 \pm 0.4$. The results are shown as red circles in Fig. 1. These results validate the idea that replacing spectroscopic reference samples with high-quality photometric reference samples is a viable approach to calibrating photometric redshift distributions via cross-correlations.

Cross-correlations convert angular clustering signal into estimates of the redshift distribution, but the error in clustering estimates varies with angular scale due to sample variance, shot noise, and survey systematics. Consequently, we test how our choice of weighting function affects the recovered redshift distribution, and how varying the radial range, particularly the inner radius, impacts our results. We consider simple power-law weighting functions $g(r)=r^{\alpha}$. We expect a priori that the optimal power will be close to the approximate power-law exponent of the correlation function itself, $\alpha \sim-0.85$. Given this expectation, we expect the primary sensitivity to the radial range $r \in\left[r_{\min }, r_{\max }\right]$ will be through the scale $r_{\min }$, which is why we focus on $r_{\min }$ in this study.

A similar analysis was performed in Schmidt et al. (2013) by looking at the effects of varying $r_{\min }$ and $r_{\max }$ on simulated galaxies. We believe each cross-correlation measurement should perform this type of analysis to determine the optimal parameters for their specific samples.

We find that there is a trade-off between bias and variance: varying the power of the weight function and the inner radius of the angular integration can significantly decrease the estimated covariance at the cost of noticeable biasing. Figs 2 and 3 summarize this trade-off. Each figure has two coloured grids showing the effect of varying the power of the weight function and the inner angular integration range on the recovered redshift distribution. In the left-hand panel, we take the overall best $\chi^{2}$ fit and plot the probability $P_{k}\left(\chi^{2}\right)$ that $\chi^{2}$ be larger than the observed value given the degrees of freedom in the analysis. When this probability is small, our recovered redshift distribution is not an acceptable fit to the data. In the right-hand panel, we plot the median error of the recovered redshift distribution as estimated from jackknife samples, median $\left(\sigma_{\phi}\right)$. For both the SDSS spectroscopic galaxy reference sample and the redMaPPer reference sample, we find that $r_{\text {min }}=0.1 \mathrm{Mpc}$ and $\alpha=-1$ provide a nearly optimal trade-off between accuracy and precision. However, we note that the specific response to variations in these parameters may differ in other samples. 


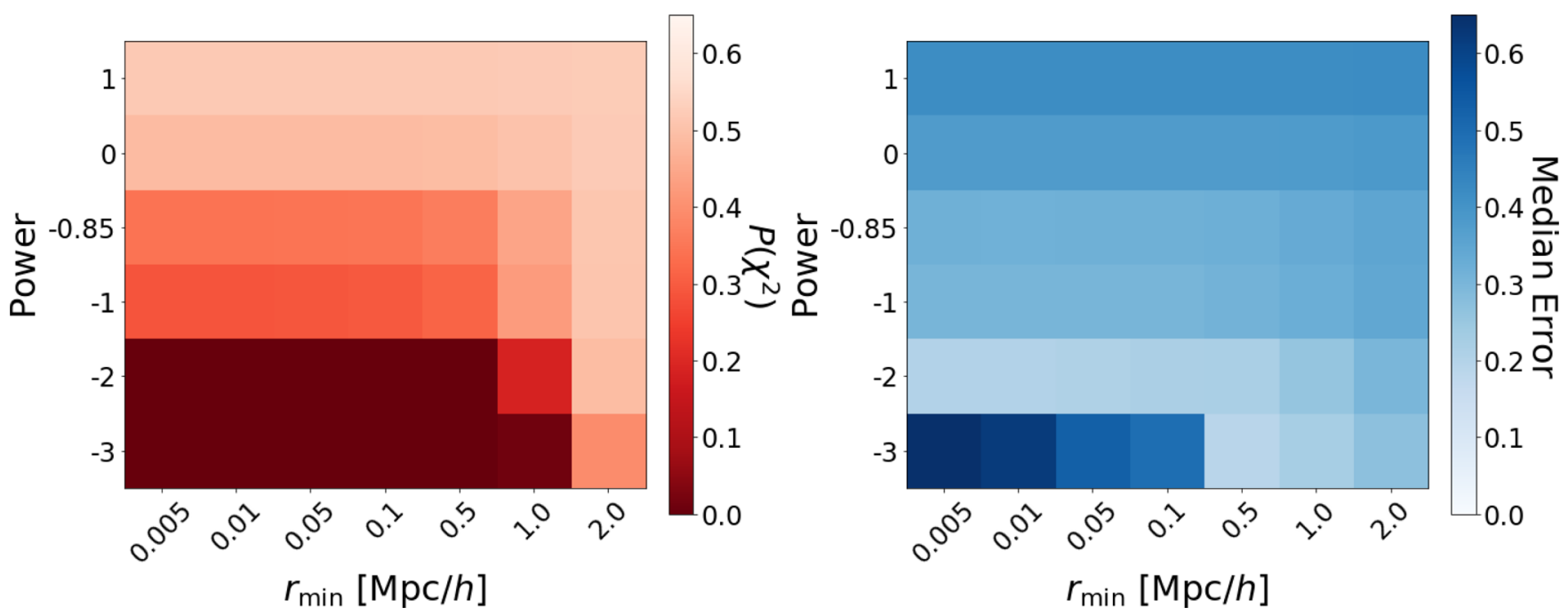

Figure 2. Effect of varying inner integration range $r_{\min }$ and weight power $\alpha$ on fit $\chi^{2}$ (left) and median error (right) for the recovery of the SDSS redshift distribution using SDSS galaxies. Setting the weight power too high will result in larger errors by emphasizing the weaker signal at larger scales, while setting the power too low will bias the measurement as extra weight is given to the non-linear regime. The power $\alpha=-0.85$ is included as a row because it is the slope of a power-law fit to the cross-correlations.

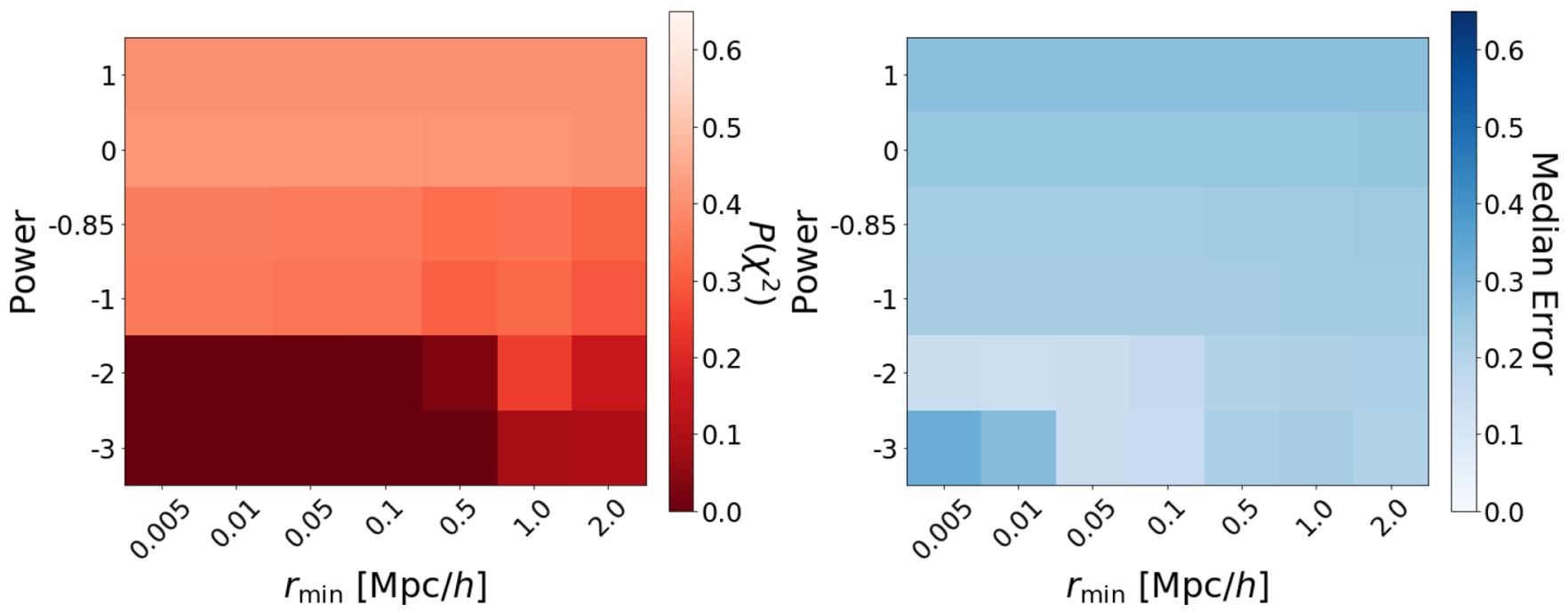

Figure 3. Effect of varying inner integration range $r_{\min }$ and weight power $\alpha$ on fit $\chi^{2}$ (left) and median error (right) for the recovery of the SDSS redshift distribution using redMaPPer galaxy clusters. As with Fig. 2, the particular choice of hyperparameters can swing the recovered distribution's fit and error properties significantly.

\section{APPLICATION TO THE DARK ENERGY SURVEY: COMBINING PHOTO-z'S WITH CROSS-CORRELATION METHODS}

It is vital for DES to accurately constrain the redshift distributions of objects that are in tomographic bins for measurements of cosmic shear or baryon acoustic oscillations. Traditionally, one places galaxies into tomographic bins based on their photometrically estimated probability distribution $P(z)$, and then one 'stacks' these $P(z)$ 's to estimate the redshift distribution of sources in a bin. The cross-correlation method as implemented here does not provide insight into which objects should go into each tomographic bin, but it does constrain the redshift distribution of the objects in each bin. Consequently, it can provide a critical systematics cross-check for photo- $z$ methods. This is especially desirable because the inputs and systematics that affect the cross-correlation method are largely independent of those affecting photo- $z$ methods. In particular, while spectroscopic redshift incompleteness is the primary difficulty affecting photo- $z$ algorithms, this systematic is completely irrelevant for cross-correlation.

In this section, we implement a cross-correlation measurement of the redshift distribution of NGMIX galaxies using redMaPPer clusters as the reference sample. The galaxies are placed into three tomographic bins with edges at $0.3,0.55,0.83,1.3$ according to the mean redshift of the SkyNet photo- $z$ code. While the NGMIX galaxies span a wide range of redshifts, we must limit our analyses to $0.2<z<0.84$, where we have redMaPPer clusters with reliable redshifts. Even with this limited redshift range, we can observe a weak signal in the third tomographic bin, albeit limited to the last few redshift bins. The weak signal leads our calibration formalism below to return our priors; we learn very little from the 

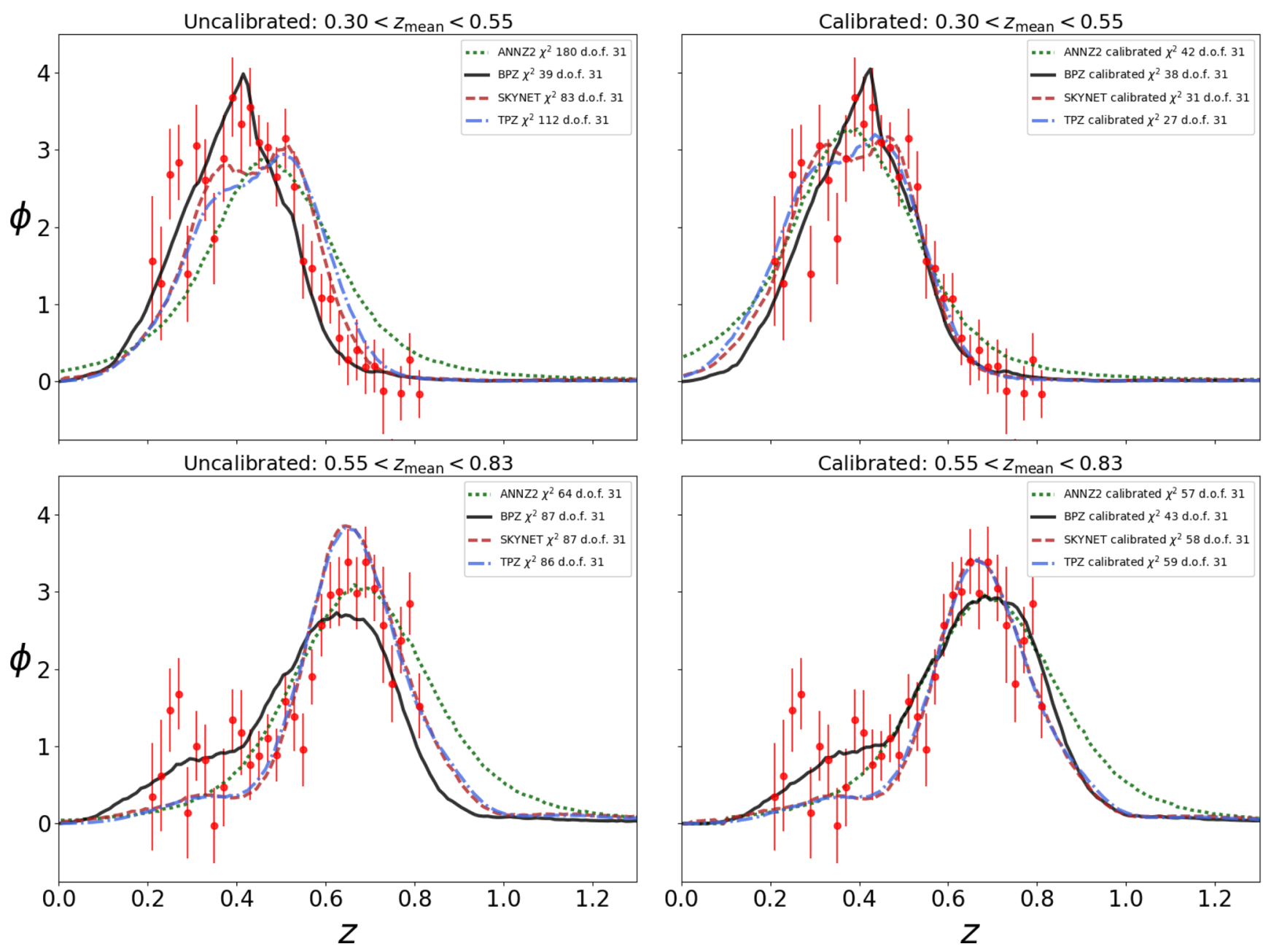

Figure 4. Redshift distribution of NGMIX galaxies using redMaPPer galaxy clusters before (left) and after (right) the calibration of equations (28) and (29). Lines represent different photo- $z$ estimation codes, while the points represent the cross-correlation method. Each row of panels represents a different tomographic bin, with galaxies selected according to the SkyNet photo-z method. Redshift distributions are normalized to one over the range of the cross-correlation sample $0.2<z<0.84$. The correction values are shown in Table 1 . Note that in practice each photo- $z$ code modifies the predicted redshift distribution from the cross-correlation analysis through the bias evolution term. In order to make it easier to visually compare the different photo- $z$ codes, we apply the corrections associated with cross-correlations to the photo- $z$ redshift distributions, which allows a direct comparison with the cross-correlation data for all photo- $z$ codes.

cross-correlations in this last bin. Therefore, we do not present the cross-correlation measurement on the third tomographic bin.

We repeat the same sort of cross-correlation analysis as in Section 4 , where we use redMaPPer clusters to measure the redshift distribution. We set the power of the weighting term, $\alpha$ to -1 and integrate from $100 \mathrm{kpc}$ to $10 \mathrm{Mpc}$. As with SDSS, we begin with the simple model in which $\gamma=0$, and the cross-correlation function is directly proportional to (and therefore an estimator of) $\phi(z)$.

Unlike in Section 4, we have no spectra that directly overlap the main DES SV footprint. Further, the fields we observed with spectra are quite small, amounting to only $\sim 10 \mathrm{deg}^{2}$. The signal from a cross-correlation analysis in this area is quite weak. Therefore, we do not check our results here against cross-correlations with spectroscopic galaxies.

We present the results of these analyses in the left column of Fig. 4. Among the four codes we consider in the two tomographic bins, only BPZ in the first tomographic bin has an acceptable $\chi^{2}$. The $\chi^{2}$ of the predicted photo- $z$ distributions relative to the recovered redshift distribution are summarized in Table 1.

We seek to reconcile photo- $z$ and cross-correlation data where possible. As discussed in Bonnett et al. (2016), the primary source
Table 1. Table of results from fitting photo- $z$ codes to cross-correlation analyses from redMaPPer clusters with DES SV NGMIX galaxies by maximizing equation (30). The 'raw' $\chi^{2}$ is the goodness of fit before allowing for an overall photometric bias of the photo- $z$ codes. The 'cal' $\chi^{2}$ value is that obtained after calibrating this bias using cross-correlations.

\begin{tabular}{lcrrrrc}
\hline Photo- $z$ & Bin & $\chi_{\text {raw }}^{2}$ & $\chi_{\text {cal }}^{2}$ & dof & \multicolumn{1}{c}{$\Delta z$} & $\gamma$ \\
\hline ANNZ2 & 1 & 180 & 42 & 31 & $0.082 \pm 0.011$ & $0.6 \pm 0.5$ \\
ANNZ2 & 2 & 64 & 57 & 31 & $-0.020 \pm 0.014$ & $0.6 \pm 0.5$ \\
BPZ & 1 & 39 & 38 & 31 & $-0.005 \pm 0.006$ & $0.1 \pm 0.4$ \\
BPZ & 2 & 87 & 43 & 31 & $-0.058 \pm 0.011$ & $0.1 \pm 0.4$ \\
SKYNET & 1 & 83 & 31 & 31 & $0.045 \pm 0.008$ & $0.5 \pm 0.5$ \\
SKYNET & 2 & 87 & 58 & 31 & $-0.018 \pm 0.007$ & $0.5 \pm 0.5$ \\
TPZ & 1 & 112 & 27 & 31 & $0.061 \pm 0.009$ & $0.5 \pm 0.5$ \\
TPZ & 2 & 86 & 59 & 31 & $-0.019 \pm 0.008$ & $0.5 \pm 0.5$ \\
\hline
\end{tabular}

of systematic uncertainty affecting the photo- $z$ distributions is an overall photometric redshift bias. We utilize our cross-correlation data to calibrate this photometric redshift bias. In practice, this means replacing the photometric redshift distribution $\phi_{\mathrm{u}}(z)$ by the 
function $\phi_{\mathrm{u}}(z-\Delta z)$, where $\Delta z$ is the photometric redshift bias of the algorithm in question. We write

$\chi^{2}=\Delta w \hat{\Sigma}^{-1} \Delta w$,

where

$\Delta w=\hat{w}-\mathrm{e}^{k}\left(\frac{1+z}{1+z_{0}}\right)^{\gamma} \phi(z-\Delta z)$.

The quantity $k$ accounts for an overall relative normalization of the distributions, since we normalize $\hat{w}(z)$ to unity within the redshift range $[0.2,0.84]$, whereas $\phi(z)$ is normalized to unity within $[0, \infty]$. We also account for clustering bias evolution from equation (24) via our parametrization of $(1+z)^{\gamma}$, which is normalized by $1+z_{0}$, where $z_{0}$ is chosen to be 0.52 , the centre of the cross-correlation redshift range. Because redshift biases and normalizations may change across tomographic bins, we define separate variables $k_{i}, \Delta z_{i}$ for each tomographic bin. In contrast, we force each tomographic bin to simultaneously fit the same $\gamma$. This is consistent with our choice to encode all redshift evolution information into $\gamma$, although we note that clustering bias evolution may be induced by the photo- $z$ selection of the NGMIX galaxies into different tomographic bins. Not surprisingly, allowing for independent $\gamma$ in each of the tomographic bins can introduce large uncertainties in the recovered distributions since any individual tomographic bin is too narrow to properly constrain the redshift-dependent evolution of the clustering bias; one really needs the full range of redshifts probed by the data.

We also utilize our prior knowledge of the redshift distributions from the photo- $z$ codes. We model our prior on the redshift bias $\Delta z_{i}$ for each tomographic bin as a Gaussian centred on 0 with a width $\sigma=0.05$ from prior analyses of photo- $z$ uncertainties from Bonnett et al. (2016). Thus, the expression we maximize is

$$
\ln \mathcal{L}=-\frac{1}{2} \chi^{2}\left(k_{i}, \Delta z_{i}, \gamma\right)-\sum_{j} \frac{1}{2} \frac{\Delta z_{j}^{2}}{2 \times 0.05^{2}} .
$$

When cross-correlations are able to strongly constrain the redshift bias (as is the case here), the particular choice of prior does not matter. In cases when cross-correlations are unable to constrain the redshift bias we simply recover our prior. By using priors informed by photo- $z$ methods, we open an avenue through which we may combine photo- $z$ and cross-correlation measurements.

We sample equation (30) using emcee, ${ }^{4}$ an affine invariant Markov Chain Monte Carlo ensemble sampler (Foreman-Mackey et al. 2013). Results of these fits are presented in the right column of Fig. 4 and in Table 1. Allowing for an overall redshift bias, all four photo- $z$ codes have an acceptable $\chi^{2}$, with TPZ resulting in the best fit. The good agreement between the codes is also apparent from Fig. 4. By contrast, only one of the codes, BPZ, has an acceptable $\chi^{2}$ for the second tomographic bin. Fig. 4 makes it apparent that most codes agree quite well on the shape of the photo- $z$ distribution in the vicinity of the average redshift of the galaxies in the bin, but differ significantly on the amplitude of a low-redshift tail in the distribution. The relatively small area of the DES SV region makes the correlation function measurements themselves very noisy in this regime, though the data does seem to indicate a preference for a larger tail, in agreement with the BPZ prediction. Future analyses from the full DES footprint should be able to clearly resolve this feature, and establish whether the 'blips' seen in the lower right-hand panel of Fig. 4 are real or just random fluctuations.
It is important to note that we also find discrepancies in our constraints on $\gamma$ from the different photo- $z$ codes. This difference is easy to explain: the shape $\phi(z)$ of the distribution is clearly degenerate with $\gamma$. Since the different photo- $z$ codes have different shapes, $\gamma$ takes on a different value for each photo- $z$ code. This demonstrates that the shape of $\phi(z)$ estimated from the traditional photo- $z$ method constitutes an important systematic for our proposed method. Future work that seeks to implement the proposed method in cosmological analyses must properly quantify the associated systematic error, for instance through the use of simulated data.

Keeping in mind the above important caveat, it is reassuring to see that for the majority of the photo- $z$ codes the posterior on the redshift bias is less than 0.05 in magnitude, consistent with the expectations of Bonnett et al. (2016). In this context, it is also worth noting that our posteriors on $\Delta z$ measure the relative redshift bias between our unknown and reference samples. redMaPPer itself has photo- $z$ redshift biases that are unconstrained at the level of $\sigma_{\Delta z} \sim 0.003$. These are currently significantly smaller than the uncertainties from cross-correlations, but may need to be accounted for as the statistics of cross-correlations improve to DES Y5 levels.

Nevertheless, it is encouraging to see that the statistical precision from this analysis in the DES SV region $\left(\sim 139 \mathrm{deg}^{2}\right)$ is not far from what would be required for a DES Y5 $\left(\sim 5000 \mathrm{deg}^{2}\right)$ cosmology analysis. Of course, in practice, detailed simulation work will be required to properly characterize the systematic uncertainties associated with this type of analysis, and possibly motivate alterations to the simple algorithm proposed here. Motivated by these results, we have launched such a study in preparation for future DES cosmological analyses.

As a final test of our proposed analysis, we revisit the SDSS data set, and test our full algorithm there by calibrating the relative redshift bias between SDSS spectroscopic galaxies and redMaPPer clusters. As expected, we find the recovered redshift bias is consistent with zero $\Delta z=(-5.1 \pm 4.6) \times 10^{-3}$, while the clustering bias evolution parameter $\gamma=-0.3 \pm 0.5$. This level of uncertainty is consistent with a priori expectations of the redMaPPer redshift bias.

\section{COSMOLOGICAL FORECASTS}

We now estimate the efficacy of cross-correlation redshift calibration for cosmological analyses of DES Y5 cosmic shear data. We start with the DES SV NGMIX cosmic shear two-point correlations. In order to perform our forecast, we must also specify a fiducial redshift distribution of sources. In keeping with the cosmic shear analysis of Becker et al. (2016), we take SkyNet as our fiducial redshift distribution. Here, we focus on the recovery of $\sigma_{8}$ (holding all other cosmological parameters fixed to their fiducial value) through the cosmology analysis pipeline used in Bonnett et al. (2016). ${ }^{5}$

We focus on two key sources of uncertainty in cosmic shear measurements: errors in the measurement of the shear two-point correlation function, and errors in our characterization of the redshift distribution from either photo- $z$ or cross-correlation systematics. We compare the posteriors in $\sigma_{8}$ for three different fiducial analyses. First, an analysis that includes only shape noise, and the source redshifts are assumed to be perfectly known. Secondly, an analysis that assumes no shape noise, but considers only the error in $\sigma_{8}$ introduced by an overall unknown photo- $z$ redshift bias with current photo- $z$ priors. Thirdly, an analysis that assumes no shape noise, but

${ }^{4}$ http://dan.iel.fm/emcee

${ }^{5}$ https://github.com/matroxel/destest 
considers the error in $\sigma_{8}$ introduced by an unknown photo- $z$ redshift bias that is calibrated using cross-correlation.

To model our DES Y5 shear covariances, we scale the DES SV cosmic shear covariances by the area of the final survey, so that we divide the DES SV cosmic shear covariance matrix by 5000/139, the ratio of DES Y5 to DES SV SPTE survey areas. Under the assumption that the galaxy density measured in SV will be equal to what we will have in Y5, this scaling is the improvement in statistical power from repeating our SV analysis over the full Y5 footprint.

To estimate the uncertainty in the recovered $\sigma_{8}$ due to an unknown photometric redshift bias with some prior and no cross-correlation data, we sample the redshift distributions $P(z-\Delta z)$ for the source galaxies, where $\Delta z$ is the redshift bias. We find the best-fitting $\sigma_{8}$ given the stacked $\mathrm{d} N / \mathrm{d} z$, and repeat our measurement 250 times, randomly drawing the redshift bias from the prior $\Delta z=0.00 \pm 0.05$. This is the level of constraint if the same photo- $z$ algorithms from DES SV were used in DES Y5, and is hence our 'conservative' case. The standard deviation in the recovered $\sigma_{8}$ values is the uncertainty in $\sigma_{8}$ due to photometric redshift uncertainties. We also consider the case where photo- $z$ algorithms improve in the measurement of systematic redshift bias to $\sigma_{\Delta z}=0.02$. We refer this as our 'optimistic' scenario. Note that 'optimistic' should not be confused with 'unrealistic'; DES Y5 data will have significantly more spectroscopic overlap over a larger area, and we expect general improvements to the data reduction pipeline in the intervening years will lead to better photometric redshift estimation. Our specific choice of $\sigma_{\Delta z}=0.02$ was achieved in Bonnett et al. (2016) for the full (non-tomographic) DES SV weak lensing sample. We caution that this level of achievement is doable, but not easy, and it will necessitate significant work on both spectroscopic and photometric calibration.

To measure the effect of cross-correlation on the recovery of $\sigma_{8}$, we begin by estimating the uncertainty in the photometric redshift bias $\sigma_{\Delta z}$ as estimated from the cross-correlation method, as per our discussion in Section 5. This constraint depends on our fit for equation (28). We scale our estimated errors $\hat{\Sigma}$ by the ratio of the survey areas between the DES Y5 and DES SV surveys (i.e. 5000/139) to simulate the increase in signal. By then setting $\hat{\phi}=\phi$ and fitting as described in Section 5, we sample the space of solutions that are consistent with the cross-correlation method. These samples provide the redshift bias $\Delta z$ that goes into our cosmology analysis. This is roughly equivalent to the simpler method of scaling the errors in the photometric redshift biases in Table 1 by the square root of the ratio of the survey areas between the DES Y5 and DES SV surveys, but has the capability for incorporating more complex modifications of the redshift distribution in the future. When comparing the optimistic and conservative cases, we use the fact that we know from the photo- $z$ algorithms that $\sigma_{\Delta z}=(0.02,0.05)$, respectively, by putting that information into our prior on the redshift bias via equation (30). This makes a significant difference because the cross-correlation cannot calibrate the highest tomographic bin due to a lack of overlap with the redMaPPer clusters, and so we revert back to the prior there. In addition, using the sampling method used to characterize the cosmological uncertainties from photo- $z$, we also add a systematic redshift error $\sigma_{\Delta z}=0.003$ to our reference sample to account for the photometric redshift bias expected for redMaPPer clusters.

Fig. 5 compares the errors in $\sigma_{8}$ from each of the three analyses considered above. We plot the ratio of the uncertainty on $\sigma_{8}$ arising from uncertainty in the mean of the redshift distribution (either from photo- $z$ or cross-correlation calibration) to the uncertainty on $\sigma_{8}$ arising from shape noise in the shear two-point correlation

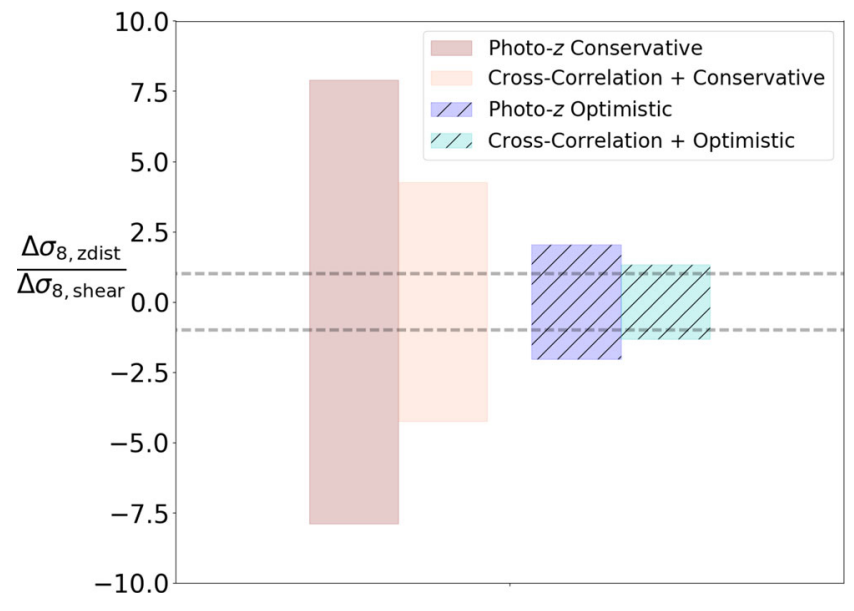

Figure 5. Ratio of the variation in $\sigma_{8}$ due to forecasted DES Y5 uncertainties from redshift distributions to shear statistical uncertainties. When this ratio is less than one, the uncertainty on $\sigma_{8}$ is dominated by errors in shape measurements; when the ratio is greater than one, errors from the redshift distribution dominate. The red (blue) band is the uncertainty due to the SkyNet photo- $z$ code with the conservative (optimistic) uncertainty $\sigma_{\Delta z}=0.05\left(\sigma_{\Delta z}=0.02\right)$ in the bias of the redshift distribution. The magenta (cyan) band is the residual uncertainty from using cross-correlations to constrain the redshift bias $\Delta z$. The grey dashed lines are guidelines for \pm 1 . Tomographic constraints are limited by the lack of overlap with redMaPPer clusters in the third (highest redshift) tomographic bin, which prevents any cross-correlation calibration in that bin.

function. When this ratio is less than one, the uncertainty on $\sigma_{8}$ is dominated by errors in shape measurements; when the ratio is greater than one, errors from the redshift distribution dominate. There is a noticeable improvement in the recovered cosmological parameters in our conservative analysis, but only a modest improvement in our optimistic scenario. This largely reflects the fact that our analyses do not constrain the highest tomographic redshift bin, which contains a large fraction of the cosmological signal. Nevertheless, it is very encouraging that even with this simple approach, and without incorporating the gains from high-redshift cross-correlation analyses that may be enabled in the future, a combined photo- $z$ and cross-correlation analysis appears to have the potential to control photometric redshift systematics. Consequently, our results provide strong motivation for further investigations into the possible use of photometric cross-correlations for photo- $z$ calibration.

\section{SUMMARY AND CONCLUSIONS}

We examined the performance of cross-correlation methods when photometric redshifts are used instead of spectroscopic galaxies as a reference sample. We first verified that cross-correlation methods work with spectroscopic reference samples by looking at the cross-correlation of SDSS galaxies. We then found that redMaPPer galaxy clusters, despite having photometric redshifts, are also viable reference samples for measuring accurate redshift distributions.

Having validated our methodology on SDSS spectroscopic galaxies, we turned towards the DES NGMIX sample in the DES SV data set. There, we applied the cross-correlation method and compared our measured redshift distributions to those measured by a variety of photo- $z$ codes. We developed a formulation for calibrating systematic redshift biases in the photo- $z$ codes, using information from both the photo- $z$ and cross-correlation methods to improve the characterization of redshift distributions. We found that the recovered redshift bias relative to the cross-correlation 
measurement is typically less than $\sim 0.05$, with the posterior of the redshift bias being uncertain at the \pm 0.01 level. This level of uncertainty is comparable to the required DES Y5 uncertainty and should only improve with five more years of data. However, systematic errors, particularly from the evolution of clustering bias in both the source and reference samples, as well as from discrepancies between the shapes of the photo- $z$ and cross-correlation redshift distributions, need to be fully characterized for this method to be integrated into future cosmological analyses.

Finally, we extrapolated the recovered statistical uncertainties to DES Y5 data, and compared the statistical uncertainty in $\sigma_{8}$ from shape noise, the photometric uncertainties from a fiducial photo- $z$ algorithm with an unknown redshift bias at the 2 percent level, and a fiducial analysis that includes calibrations from crosscorrelations. The impact of the cross-correlation analysis depends on the uncertainty that traditional photo- $z$ algorithm can reach in the near future. Even in our optimistic scenario, however, crosscorrelations provide a non-negligible improvement in the recovered cosmological constraints, while simultaneously providing a critical consistency test of the photo- $z$ calibration.

These results firmly establish the feasibility of using photometric samples as reference samples for calibrating photometric redshift distributions via cross-correlation, and provide strong motivation for more detailed simulation-based studies aimed at fully characterizing the systematic uncertainties in these methods. In particular, we have already seen hints that systematic uncertainties in the shape of the $\phi(z)$ estimated from traditional photo- $z$ methods can introduce systematics in the recovered photometric redshift bias. We are currently characterizing these systematics via numerical simulations for the DES Year 1 cosmology analysis (Davis et al. 2017; Gatti et al. 2017). Similarly, it is not difficult to imagine modifications of the methodology adopted here that is more ideally suited to cosmological analyses. For example, cosmic shear analyses are primarily sensitive to photo- $z$ uncertainties through the photometric redshift bias and not the shape of the redshift distribution. The methods presented here could be tailored towards these goals by matching the means of the distributions while down-weighting the tails of the redshift distributions, thereby minimizing systematics associated with the detailed shape of $\phi(z)$. We are also exploring alternative high-fidelity photometric reference samples such as the luminous red galaxy redMaGiC sample described in Rozo et al. (2016), which has considerably higher number density than redMaPPer clusters (Cawthon et al. 2017). The fact that the cross-correlation provides a calibration tool that is demonstrably capable of reaching the necessary statistical precision for Stage III dark energy experiments is an important step forward in realizing the promise of the DES, and provides strong motivation for further studies of this method.

\section{ACKNOWLEDGEMENTS}

CPD would like to acknowledge Enrique Gaztanaga, Marco Gatti, Pauline Vielzeuf, Ross Cawthon, and Àlex Alarcón for many fruitful conversations. He would also like to thank Ben Hoyle, Huan Lin, Ramon Miquel, and Michael Troxel for their many suggestions, which tremendously improved this paper. This work is partially supported by the Northern California Chapter of the ARCS Foundation, as well as by the U.S. Department of Energy under contract number DE-AC02-76-SF00515. ER is supported by DOE grant DESC0015975 and by the Sloan Foundation, grant FG-2016-6443.

Funding for the DES Projects has been provided by the U.S. Department of Energy, the U.S. National Science Foundation, the Ministry of Science and Education of Spain, the Science and
Technology Facilities Council of the United Kingdom, the Higher Education Funding Council for England, the National Center for Supercomputing Applications at the University of Illinois at UrbanaChampaign, the Kavli Institute of Cosmological Physics at the University of Chicago, the Center for Cosmology and Astro-Particle Physics at the Ohio State University, the Mitchell Institute for Fundamental Physics and Astronomy at Texas A\&M University, Financiadora de Estudos e Projetos, Fundação Carlos Chagas Filho de Amparo à Pesquisa do Estado do Rio de Janeiro, Conselho Nacional de Desenvolvimento Científico e Tecnológico and the Ministério da Ciência, Tecnologia e Inovação, the Deutsche Forschungsgemeinschaft, and the Collaborating Institutions in the DES.

The Collaborating Institutions are Argonne National Laboratory, the University of California at Santa Cruz, the University of Cambridge, Centro de Investigaciones Energéticas, Medioambientales y Tecnológicas-Madrid, the University of Chicago, University College London, the DES-Brazil Consortium, the University of Edinburgh, the Eidgenössische Technische Hochschule (ETH) Zürich, Fermi National Accelerator Laboratory, the University of Illinois at Urbana-Champaign, the Institut de Ciències de 1'Espai (IEEC/CSIC), the Institut de Física d'Altes Energies, Lawrence Berkeley National Laboratory, the Ludwig-Maximilians Universität München and the associated Excellence Cluster Universe, the University of Michigan, the National Optical Astronomy Observatory, the University of Nottingham, The Ohio State University, the University of Pennsylvania, the University of Portsmouth, SLAC National Accelerator Laboratory, Stanford University, the University of Sussex, Texas A\&M University, and the OzDES Membership Consortium.

The DES data management system is supported by the National Science Foundation under grant numbers AST-1138766 and AST-1536171. The DES participants from Spanish institutions are partially supported by MINECO under grants AYA201571825, ESP2015-88861, FPA2015-68048, SEV-2012-0234, SEV2012-0249, and MDM-2015-0509, some of which include ERDF funds from the European Union. IFAE is partially funded by the CERCA program of the Generalitat de Catalunya.

We are grateful for the extraordinary contributions of our CTIO colleagues and the DECam Construction, Commissioning and Science Verification teams in achieving the excellent instrument and telescope conditions that have made this work possible. The success of this project also relies critically on the expertise and dedication of the DES Data Management group.

\section{REFERENCES}

Alam S. et al., 2015, ApJS, 219, 12

Asorey J., Carrasco Kind M., Sevilla-Noarbe I., Brunner R. J., Thaler J., 2016, MNRAS, 459, 1293

Becker M. R. et al., 2016, Phys. Rev. D, 94, 022002

Bell E. F. et al., 2004, ApJ, 608, 752

Benítez N., 1999, in Weymann R., Storrie-Lombardi L., Sawicki M., Brunner R., eds, ASP Conf. Ser. Vol. 191, Photometric Redshifts and the Detection of High Redshift Galaxies. Astron. Soc. Pac., San Francisco, p. 31

Benjamin J., van Waerbeke L., Ménard B., Kilbinger M., 2010, MNRAS, 408,1168

Bertin E., 2010, SWarp: Resampling and Co-adding FITS Images Together, Astrophysics Source Code Library, record ascl:1010.068

Bertin E., 2011, in Evans I. N., Accomazzi A., Mink D. J., Rots A. H., eds, ASP Conf. Ser. Vol. 442, Astronomical Data Analysis Software and Systems XX. Astron. Soc. Pac., San Francisco, p. 435

Bertin E., Arnouts S., 1996, A\&AS, 117, 393 
Bonnett C., 2015, MNRAS, 449, 1043

Bonnett C. et al., 2016, Phys. Rev. D, 94, 042005

Bower R. G., Lucey J. R., Ellis R. S., 1992, MNRAS, 254, 601

Carlstrom J. E. et al., 2011, PASP, 123, 568

Carrasco Kind M., Brunner R., 2013, TPZ: Trees for Photo-Z, Astrophysics Source Code Library, record ascl:1304.011

Carrasco Kind M., Brunner R., 2014, MLZ: Machine Learning for Photo-Z, Astrophysics Source Code Library, record ascl:1403.003

Cawthon R. et al., 2017, MNRAS, preprint (arXiv:1712.07298)

Choi A. et al., 2016, MNRAS, 463, 3737

Coe D., Benítez N., Sánchez S. F., Jee M., Bouwens R., Ford H., 2006, AJ, 132,926

Cunha C. E., Lima M., Oyaizu H., Frieman J., Lin H., 2009, MNRAS, 396, 2379

Davis C. et al., 2017, MNRAS, preprint (arXiv:1710.02517)

Desai S. et al., 2012, ApJ, 757, 83

Flaugher B. et al., 2015, AJ, 150, 150

Foreman-Mackey D., Hogg D. W., Lang D., Goodman J., 2013, PASP, 125, 306

Gatti M. et al., 2017, MNRAS, preprint (arXiv:1709.00992v1)

Graff P., Feroz F., Hobson M. P., Lasenby A., 2014, MNRAS, 441, 1741

Hildebrandt H. et al., 2017, MNRAS, 465, 1454

Hinshaw G. et al., 2013, ApJS, 208, 19

Hogg D. W., Lang D., 2013, PASP, 125, 719

Jarvis M., Bernstein G., Jain B., 2004, MNRAS, 352, 338

Jarvis M. et al., 2016, MNRAS, 460, 2245

Johnson A. et al., 2017, MNRAS, 465, 4118

Landy S. D., Szalay A. S., 1993, ApJ, 412, 64

Lee B. C. G., Budavári T., Basu A., Rahman M., 2016, AJ, 152, 155

Lima M., Cunha C. E., Oyaizu H., Frieman J., Lin H., Sheldon E. S., 2008, MNRAS, 390, 118

Matthews D. J., Newman J. A., 2010, ApJ, 721, 456

Matthews D. J., Newman J. A., 2012, ApJ, 745, 180

McLeod M., Balan S. T., Abdalla F. B., 2017, MNRAS, 466, 3558

McQuinn M., White M., 2013, MNRAS, 433, 2857

Ménard B., Scranton R., Schmidt S., Morrison C., Jeong D., Budavari T., Rahman M., 2013, preprint (arXiv:1303.4722)

Morganson E. et al., 2018, preprint (arXiv:1801.03177)

Morrison C. B., Hildebrandt H., Schmidt S. J., Baldry I. K., Bilicki M., Choi A., Erben T., Schneider P., 2017, MNRAS, 467, 3576

Newman J. A., 2008, ApJ, 684, 88

Rahman M., Ménard B., Scranton R., Schmidt S. J., Morrison C. B., 2015, MNRAS, 447, 3500

Rahman M., Mendez A. J., Ménard B., Scranton R., Schmidt S. J., Morrison C. B., Budavári T., 2016, MNRAS, 460, 163

Rozo E., Rykoff E. S., Bartlett J. G., Melin J.-B., 2015a, MNRAS, 450, 592

Rozo E., Rykoff E. S., Becker M., Reddick R. M., Wechsler R. H., 2015b, MNRAS, 453, 38

Rozo E. et al., 2016, MNRAS, 461, 1431

Rykoff E. S. et al., 2014, ApJ, 785, 104

Rykoff E. S. et al., 2016, ApJS, 224, 1

Sadeh I., Abdalla F. B., Lahav O., 2016, PASP, 128, 104502

Sánchez C. et al., 2014, MNRAS, 445, 1482

Schmidt S. J., Ménard B., Scranton R., Morrison C., McBride C. K., 2013, MNRAS, 431, 3307

Schmidt S. J., Ménard B., Scranton R., Morrison C. B., Rahman M., Hopkins A. M., 2015, MNRAS, 446, 2696

Schneider M., Knox L., Zhan H., Connolly A., 2006, ApJ, 651, 14

Schulz A. E., 2010, ApJ, 724, 1305

Scottez V. et al., 2016, MNRAS, 462, 1683

Scottez V., Benoit-Lévy A., Coupon J., Ilbert O., Mellier Y., 2018, MNRAS, 474, 3921

Sevilla I. et al., 2011, preprint (arXiv:1109.6741)

Sheldon E. S., 2014, MNRAS, 444, L25

Sun L., Zhan H., Tao C., 2015, preprint (arXiv:1512.00600v1)

van Daalen M. P., White M., 2017, MNRAS, 476, 4649

Zuntz J., Kacprzak T., Voigt L., Hirsch M., Rowe B., Bridle S., 2013, MNRAS, 434, 1604
${ }^{1}$ Kavli Institute for Particle Astrophysics and Cosmology, PO Box 2450, Stanford University, Stanford, CA 94305, USA

${ }^{2}$ Institute of Space Sciences, IEEC-CSIC, Campus UAB, Carrer de Can Magrans, s/n, E-08193 Barcelona, Spain

${ }^{3}$ Kavli Institute for Cosmological Physics, University of Chicago, Chicago, IL 60637, USA

${ }^{4}$ Institut de Fisica d'Altes Energies (IFAE), The Barcelona Institute of Science and Technology, Campus UAB, E-08193 Bellaterra (Barcelona), Spain

${ }^{5}$ Fermi National Accelerator Laboratory, PO Box 500, Batavia, IL 60510, USA

${ }^{6}$ Institució Catalana de Recerca i Estudis Avançats, E-08010 Barcelona, Spain

${ }^{7}$ SLAC National Accelerator Laboratory, Menlo Park, CA 94025, USA

${ }^{8}$ Department of Physics, University of Arizona, Tucson, AZ 85721, USA

${ }^{9}$ Center for Cosmology and Astro-Particle Physics, The Ohio State University, Columbus, OH 43210, USA

${ }^{10}$ Department of Physics, The Ohio State University, Columbus, $\mathrm{OH} 43210$, USA

${ }^{11}$ Cerro Tololo Inter-American Observatory, National Optical Astronomy Observatory, Casilla 603, La Serena, Chile

${ }^{12}$ Department of Physics and Astronomy, University College London, Gower Street, London WC1E 6BT, UK

${ }^{13}$ Department of Physics and Electronics, Rhodes University, PO Box 94 , Grahamstown 6140, South Africa

${ }^{14}$ LSST, 933 North Cherry Avenue, Tucson, AZ 85721, USA

${ }^{15}$ CNRS, UMR 7095, Institut d'Astrophysique de Paris, F-75014 Paris,

France

${ }^{16}$ Sorbonne Universités, UPMC Univ Paris 06, UMR 7095, Institut d'Astrophysique de Paris, F-75014 Paris, France

${ }^{17}$ Laboratório Interinstitucional de e-Astronomia - LIneA, Rua Gal. José Cristino 77, Rio de Janeiro RJ-20921-400, Brazil

${ }^{18}$ Observatório Nacional, Rua Gal. José Cristino 77, Rio de Janeiro RJ20921-400, Brazil

${ }^{19}$ Department of Astronomy, University of Illinois, 1002 W. Green Street, Urbana, IL 61801, USA

${ }^{20}$ National Center for Supercomputing Applications, 1205 West Clark St., Urbana, IL 61801, USA

${ }^{21}$ Department of Physics and Astronomy, University of Pennsylvania, Philadelphia, PA 19104, USA

${ }^{22}$ Department of Physics, IIT Hyderabad, Kandi, Telangana 502285, India

${ }^{23}$ Instituto de Fisica Teorica UAM/CSIC, Universidad Autonoma de Madrid, E-28049 Madrid, Spain

${ }^{24}$ Department of Astronomy, University of Michigan, Ann Arbor, MI 48109, USA

${ }^{25}$ Department of Physics, University of Michigan, Ann Arbor, MI 48109 , USA

${ }^{26}$ Institute of Astronomy, University of Cambridge, Madingley Road, Cambridge CB3 OHA, UK

${ }^{27}$ Kavli Institute for Cosmology, University of Cambridge, Madingley Road, Cambridge CB3 OHA, UK

${ }^{28}$ Universitäts-Sternwarte, Fakultät für Physik, Ludwig-Maximilians Universität München, Scheinerstr. 1, D-81679 München, Germany

${ }^{29}$ Astronomy Department, University of Washington, Box 351580, Seattle, WA 98195, USA

${ }^{30}$ Santa Cruz Institute for Particle Physics, Santa Cruz, CA 95064, USA

${ }^{31}$ Australian Astronomical Observatory, North Ryde, NSW 2113, Australia

${ }^{32}$ Argonne National Laboratory, 9700 South Cass Avenue, Lemont, IL 60439, USA

${ }^{33}$ Departamento de Física Matemática, Instituto de Física, Universidade de São Paulo, CP 66318, São Paulo, SP 05314-970, Brazil

${ }^{34}$ George P. and Cynthia Woods Mitchell Institute for Fundamental Physics and Astronomy, and Department of Physics and Astronomy, Texas A\&M University, College Station, TX 77843, USA

${ }^{35}$ Department of Astronomy, The Ohio State University, Columbus, $\mathrm{OH}$ 43210, USA

${ }^{36}$ Department of Astrophysical Sciences, Princeton University, Peyton Hall, Princeton, NJ 08544, USA 
${ }^{37}$ Jet Propulsion Laboratory, California Institute of Technology, 4800 Oak Grove Dr., Pasadena, CA 91109, USA

${ }^{38}$ Department of Physics and Astronomy, Pevensey Building, University of Sussex, Brighton BN1 9QH, UK

${ }^{39}$ Centro de Investigaciones Energéticas, Medioambientales y Tecnológicas (CIEMAT), Av. Complutense 40, 28040 Madrid, Spain

${ }^{40}$ School of Physics and Astronomy, University of Southampton, Southampton $\mathrm{SO} 17$ 1BJ, UK

${ }^{41}$ Instituto de Física Gleb Wataghin, Universidade Estadual de Campinas, 13083-859 Campinas, SP, Brazil
${ }^{42}$ Computer Science and Mathematics Division, Oak Ridge National Laboratory, Oak Ridge, TN 37831, USA

${ }^{43}$ Institute of Cosmology and Gravitation, University of Portsmouth, Portsmouth PO1 3FX, UK

${ }^{44}$ Department of Physics, Stanford University, 382 Via Pueblo Mall, Stanford, CA 94305, USA

This paper has been typeset from a $\mathrm{T}_{\mathrm{E}} \mathrm{X} / \mathrm{L} \mathrm{T}_{\mathrm{E}} \mathrm{X}$ file prepared by the author. 